The Nepali Math. Sc. Report

Vol. 36, No.1 and 2, 2019

\title{
EXPERIMENTAL VALIDATION OF A MICROSCOPIC ELLIPSOIDAL INTERACTING PARTICLE MODEL IMMERSED IN FLUID FLOW
}

\author{
A. MEURER ${ }^{1}$, A. WEBER ${ }^{2}$, H.-J. BART ${ }^{2}$, A. KLAR $^{1}$, S. TIWARI ${ }^{1}$ \\ 1 Technische Universität Kaiserslautern, Department of Mathematics, \\ Erwin-Schrödinger-Straße, 67663 Kaiserslautern, Germany \\ \{meurer,klar,tiwari\}@mathematik.uni-kl.de \\ 2 Technische Universität Kaiserslautern, Department of Process Engineering, \\ Erwin-Schrödinger-Straße, 67663 Kaiserslautern, Germany \\ $\{$ andreas.weber,bart\}@mv.uni-kl.de
}

\begin{abstract}
We consider a simplified model for the simulation of suspended ellipsoidal particles in fluid flow presented in [1] and investigate the calibration of the model from lab size experiments. Data have been recorded using a camera set-up and post-processing of the pictures. The model uses a simplified description for the orientation and position of the particles based on Jeffery's equation. Additionally, particle-particle interaction and particle-wall interaction are taken into account.
\end{abstract}

Key Words: Ellipsoidal particles; Jeffery's equation; CFD simulation; experimental validation; immersed rigid body

AMS (MOS) Subject Classification. 35Kxx, 35Dxx, 65Mxx, 70Exx.

\section{INTRODUCTION}

In many industrial applications the simulation of the motion of particles suspended in a fluid is required. In the present work we consider non-spherical, ellipsoidal particles with particle-particle and particle-wall collisions. We describe the movement of ellipsoidal particles in a fluid using a simplified Langevin approach, see [1]. This means we use a system of stochastic differential equations based on Newtonian laws of mechanics and stochastic terms and calibrate the model with experimental data. To model the forces acting on the particles in the fluid, we use the model of Jeffery $[5,12,7]$. While spherical particles allow for a simple calculation of the forces acting on them, calculating the forces acting on deformed particles is more complicated, compare $[17,18]$. Here, the particle-particle interaction of the ellipses are described via pairwise interaction potentials and a random force. The potentials we use are common in the literature of polymers $[10,6,3,9,13,8]$, where the shape of the ellipses are modeled with the help of Gaussian type functions. This leads to a model similar to the one described in $[15,2,14,7]$. For macroscopic approximations of this particle model, see [1]. We note that for the applicability to a wider range of industrial applications such 
as simulation of bubbles inside chemical reactors or treatment/classification of grains, see $[19,20]$ an expanded version of the basic model discussed here would be required.

The paper is organized as follows. In section 2, we describe the lab experiment, where a channel has been filled with water and ellipsoidal particles. A camera system has been used to evaluate different parameters of the particle flow. With a suitable image analysis software we determine movement speed, orientation and residence density of the considered particles. In section 3, a CFD simulation of the fluid flow inside the channel is investigated and coupled to the simulation of the interacting ellipsoidal particle model in section 4 . Further, in section 4, the model is calibrated with data from lab experiment. Finally, the calibrated model is compared to the experimental data in section 5.

\section{EXPERIMENT}

We consider an acrylic glass channel with measures as shown in Figure 1. The channel is completely filled with reverse osmosis water, air bubbles at the lid were carefully removed. In- and outflow are connected to a pumping system which gives a constant flow of $\dot{V}=$
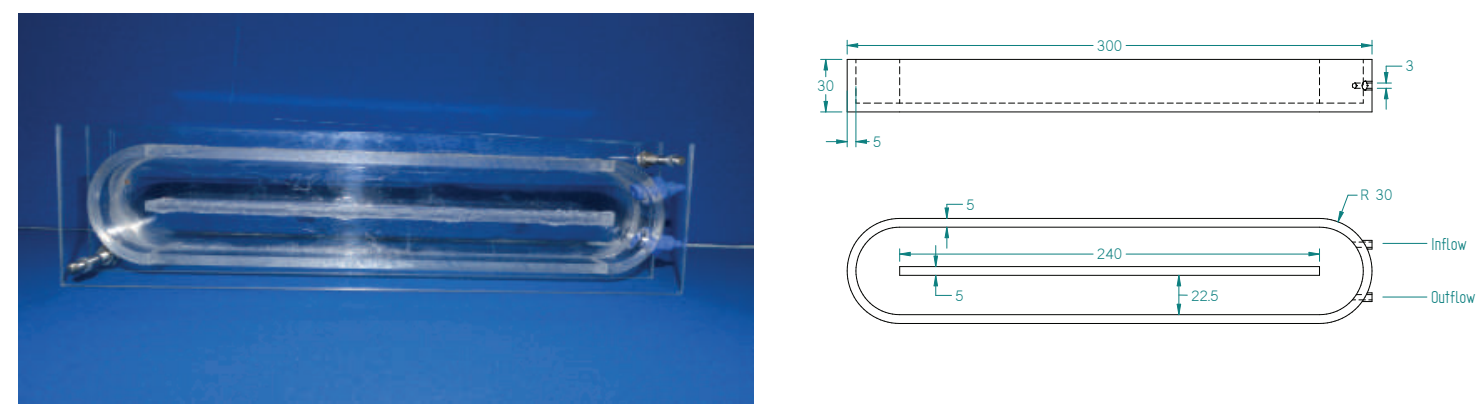

Figure 1. Picture of the acrylic glass channel on the left and the corresponding measures (in $\mathrm{mm}$ ) on the right.

$66.24 \frac{l}{h}$. At the inflow cross section of $A=9.2 \mathrm{~mm}^{2}$ an average fluid velocity of $u=2 \frac{\mathrm{m}}{\mathrm{s}}$ is reached. The water flows in at the top channel and forms a circular stream in counterclockwise direction with a mean fluid velocity of $u_{\text {mean }}=0.18 \frac{\mathrm{m}}{\mathrm{s}}$. This results in a channel Reynolds number of $R e=4350$, which implies a flow in the transitional domain. The particles added to the flow resemble prolate rotational spheroids with a major radius of $L=4.9 \mathrm{~mm}$ and minor radius of $D=2.5 \mathrm{~mm}$ as shown in Figure 2. Due to their density of $\rho=0.95 \frac{\mathrm{g}}{\mathrm{cm}^{3}}$ the particles float slightly inside the water with $\rho=1 \frac{\mathrm{g}}{\mathrm{cm}^{3}}$. Particles have been tested individually and in an ensemble of $N=25$ particles to detect the orientation and position inside the channel. Since the flow inside the pump and channel can not be switched on instantaneously, we start the detection of particles when the steady-state flow is reached. We detect the particle motion for $T=10 \mathrm{~s}$ with a frame rate of $\mathrm{fps}=25 \mathrm{~s}^{-1}$. The mean particle velocity in the channel is $v_{\text {mean }}=0.09 \frac{\mathrm{m}}{\mathrm{s}}$ which gives a particle Reynolds number of $R e_{p}=436$, implying a laminar-turbulent transitional flow around the particles. 

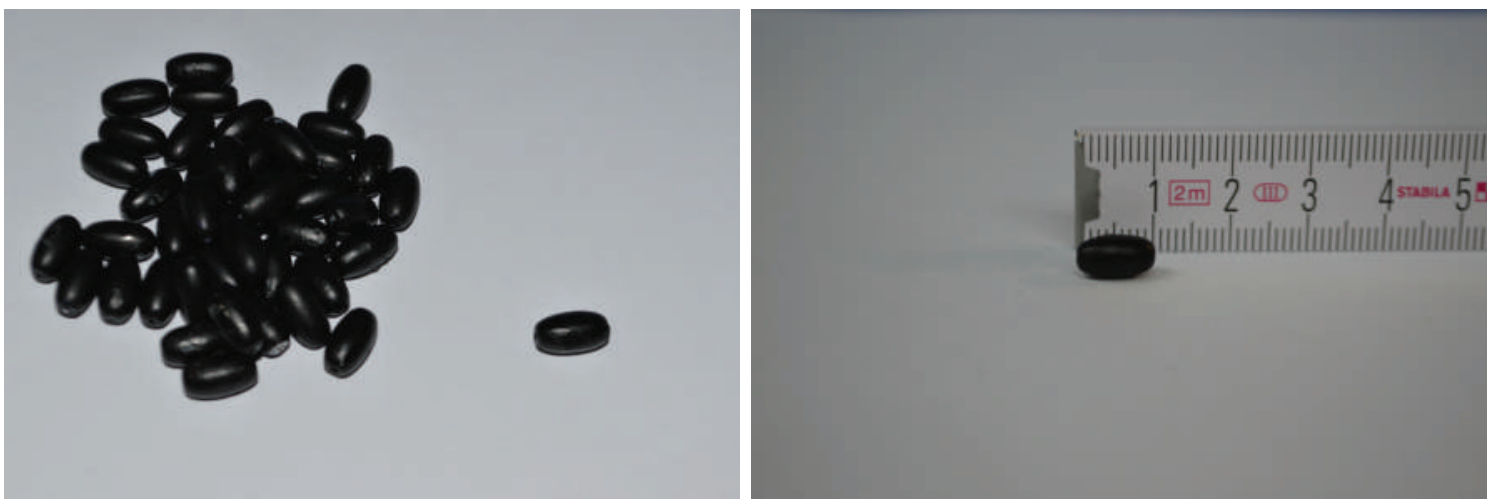

FiguRE 2. The particles which are used for the experiment.

2.1. Post-processing. In the post-processing step we use the resulting pictures to analyze the particle information with the help of the program ImageJ [16]. Since the particles have a dark color, it is possible to separate them with a threshold binarization technique. In a second step a watershed algorithm separates particles which are in direct contact and would be recognized as one larger particle otherwise. At last ImageJ measures the position, orientation, size and boundary line of each particle. This embodies the major and minor axis lengths and the angle of the major axis compared to the picture coordinate system. In total we obtain the spatial and directional distribution and the velocity of the particles. Another

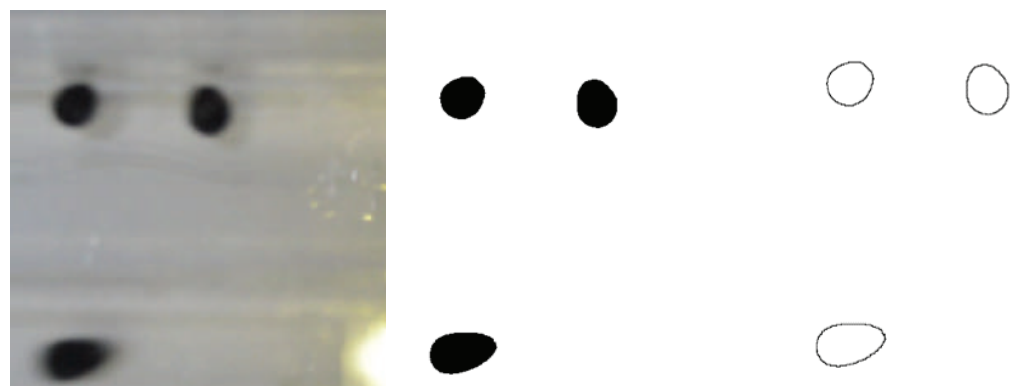

FIGURE 3. Stepwise post-processing of a picture in Image J

approach consists in utilizing the standard deviation of the pictures. An area of the channel, where it is not possible to find a particle would always show the same color/brightness on every picture. Particles increase the deviation of the brightness the more often they pass a pixel. Hence the deviation of brightness values also gives the spatial distribution of the particles. This can also be done with or without binarizing the pictures at first.

Figure 4 shows resulting deviation pictures, where darker areas stand for higher residence probability of a particle. On the left picture, note the clearly visible single particle trajectory and position of wall contact. Also the particle velocity can be deduced from the pattern of the trajectory. Here the particle is much faster in the upper channel than it is in the lower one.

The right picture was calculated from a multiple particle experiment. It is clearly visible that particles are not scattered uniformly but have a higher probability to be found near the walls of the channel. In the upper channel they are concentrated at the outer wall 

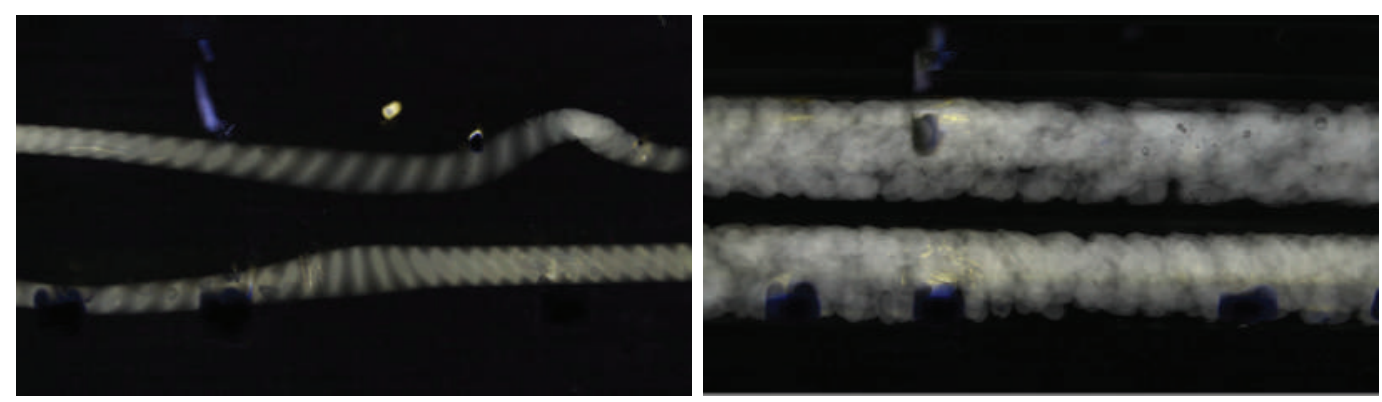

FiguRE 4. Brightness deviation of pictures from single and multiple particle experiments

while in the lower channel they appear to be more present at the inner wall. The lighter spots in the picture are a result of measurement errors, that originate from light reflections on the lid of the channel.

\section{CFD simulation}

A CFD simulation for incompressible laminar flow was used to calculate the flow pattern inside the channel. The fluid velocity field obtained from this simulation was then used for the particle simulation, see the next section. We note that measurements have been solely made on the middle part of the channel, the corners have not been part of any experimental evaluation. Thus, for simplicity, the CFD domain was chosen rectangular. In $\mathrm{cm}$ the fluid domain is given by

$$
\Omega_{F}=\left[\begin{array}{ll}
0 & 29
\end{array}\right] \times\left[\begin{array}{ll}
0 & 5
\end{array}\right] \times\left[\begin{array}{ll}
0 & 2.5
\end{array}\right] \backslash\left[\begin{array}{ll}
2.5 & 26.5
\end{array}\right] \times\left[\begin{array}{lll}
2.25 & 2.75
\end{array}\right] \times\left[\begin{array}{ll}
0 & 2.5
\end{array}\right] .
$$

For the boundary conditions we choose

- Inflow velocity of $2 \mathrm{~m} / \mathrm{s}$ in $\Omega_{I N}=\{29\} \times\left[\begin{array}{ll}3.7 & 0.4\end{array}\right] \times\left[\begin{array}{ll}1.1 & 1.4\end{array}\right]$.

- Free outflow at $\Omega_{O U T}=\{29\} \times\left[\begin{array}{ll}0 & 2.25\end{array}\right] \times\left[\begin{array}{ll}0 & 2.25\end{array}\right]$.

- No-slip condition for the other boundaries.

Transport properties were set to standard values of pure water, namely incompressible fluid with density $\rho=1000 \frac{\mathrm{kg}}{\mathrm{m}^{3}}$ and viscosity $\nu=10^{-6} \frac{\mathrm{m}^{2}}{\mathrm{~s}}$.

A mesh with 325250 cells has been used to represent the domain, giving a resolution of approximately $1 \mathrm{~mm}^{3}$ per cell. The simulation has been carried out using a laminar scheme. The typical flow pattern is shown in Figure 5. The high inflow velocity causes the flow to fluctuate inside the upper channel. The flow data used in the particle simulation were smoothed by averaging the velocity field over $\Delta t=20 \mathrm{~s}$. Since the inflow velocity is rather high, a clearly developed eddy shows up in the upper channel. In this area the fluid can move against the overall counter-clockwise direction eventually dragging particles with it. This behavior could be confirmed in the experiment (and simulation). The lower channel shows a smoother velocity profile. Only a small eddy is present directly behind the bend. The outflow in the lower right corner of the channel shows no significant influence on the flow pattern. 


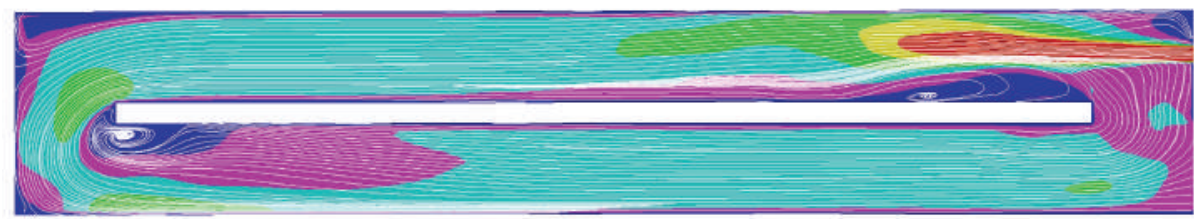

U Magnitude in $(\mathrm{m} / \mathrm{s})$

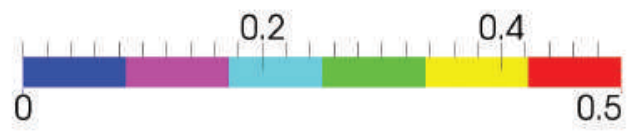

Figure 5. CFD simulation results showing fluid velocity and streamlines.

The CFD simulation was solved for the 3D case whereas for the particle model a 2D microscopic system is used, see the next section. Therefore, CFD-data had to be converted to the $2 \mathrm{D}$ model. This was done by mapping a plane through the middle of the computational domain. Fluid velocities in z-direction were small enough to be neglected, thus the resulting $2 \mathrm{D}$ velocity field is a good approximation of the real flow pattern.

\section{The Model}

To describe the movement of the ellipsoidal particles suspended in an incompressible fluid, we consider a microscopic Langevin-type model as in [7]. We use a basic twodimensional model with minimal number of forces and few assumptions. The interaction of the fluid with the ellipses is described by a Jeffery's type term $[5,12]$ and the particle interaction is given by a many-particle interaction potential similar to [6]. Each particle is described by its position $r_{t} \in \mathbb{R}^{2}$, velocity $v_{t} \in \mathbb{R}^{2}$, orientation angle $\theta_{t} \in[0,2 \pi)$ and angular velocity $\omega_{t} \in \mathbb{R}$. The angle $\theta_{t}$ describes the relative angle between the horizontal axis and the main axis of the particle, such that $\theta_{t}=0$ corresponds to the orientation $(1,0)^{T}$. Then, the equation of motion for $N$ particles $i=1, \ldots, N$ are

$$
\left\{\begin{aligned}
d r_{t}^{i}=v_{t}^{i} d t & \\
d v_{t}^{i}=\gamma\left(u-v_{t}^{i}\right) d t-\frac{1}{m} \frac{1}{N} \sum_{i \neq j} \nabla_{r_{i}} U\left(r_{t}^{i}, r_{t}^{j}, \theta_{t}^{i}, \theta_{t}^{j}\right) d t & \quad-\left(A^{2} / 2\right) v_{t}^{i} d t+A d W_{t}^{A, i} \\
d \theta_{t}^{i}=\omega_{t}^{i} d t & -\left(B^{2} / 2\right) \omega_{t}^{i} d t+B d W_{t}^{B, i},
\end{aligned}\right.
$$

with appropriate initial conditions. Here $u$ is the velocity of a surrounding fluid computed by the CFD simulation described in the last section. For the surrounding fluid, we assume 
that the influence of the particle to the fluid is negligible. The function $g(\theta, u)$ is given by

$$
g(\theta, u)=\frac{1}{2} \operatorname{curl}(u)+\lambda\left(\begin{array}{r}
-\sin \theta \\
\cos \theta
\end{array}\right)^{\top}\left(\frac{1}{2}\left(\nabla u+\nabla u^{\top}\right)\right)\left(\begin{array}{c}
\cos \theta \\
\sin \theta
\end{array}\right) .
$$

The first terms on the right hand side of the velocity and angular velocity equations describe the relaxation of the particles to the velocity of the fluid and to the rotation resulting from the velocity field, respectively. The speed of relaxation is determined by the friction parameters $\gamma$ and $\bar{\gamma}$. The second term models the repulsive interaction between the particles. To model the interaction between two ellipsoidal particles, there exist many different potentials $[10,6,3,9,13,8]$. We use the soft potential as proposed by Berne [6]. It is obtained by overlapping two ellipsoidal Gaussians representing the mutual repulsion of two particles. This leads to

$$
\tilde{U}(r, \bar{r}, \theta, \bar{\theta})=a(\theta, \bar{\theta}) \exp \left(-(\bar{r}-r)(\gamma(\theta)+\gamma(\bar{\theta}))^{-1}(\bar{r}-r)\right),
$$

where $a$ and $\gamma$ are defined by

$$
\begin{gathered}
a(\theta, \bar{\theta})=\epsilon_{0}\left(1-\lambda^{2}(\eta(\theta) \cdot \eta(\bar{\theta}))^{2}\right)^{-\frac{1}{2}}, \quad \eta(\theta)=(\cos \theta, \sin \theta)^{\top}, \\
\gamma(\theta)=\left(l^{2}-d^{2}\right) \eta(\theta) \otimes \eta(\bar{\theta})+d^{2} \nVdash, \quad \lambda=\frac{l^{2}-d^{2}}{l^{2}+d^{2}} .
\end{gathered}
$$

Here, $l=2 L$ and $d=2 D$ where $L$ is the major and the $D$ the minor radius of the particle. The parameter $\epsilon_{0}$ models the strength of the potential. To have compact support we slightly modify the potential and define

$$
U(r, \bar{r}, \theta, \bar{\theta})=a(\theta, \bar{\theta}) \exp \left(-\frac{(\bar{r}-r)(\gamma(\theta)+\gamma(\bar{\theta}))^{-1}(\bar{r}-r)}{1-(\bar{r}-r)(\gamma(\theta)+\gamma(\bar{\theta}))^{-1}(\bar{r}-r)}\right) .
$$

The parameters $m$ and $I_{c}$ are the mass and the moment of inertia of the particles. Furthermore, $A, B$ are non-negative diffusion constants and $W^{A, i}, W^{B, i}$ are independent standard Brownian motions.

4.1. Numerical Set Up. We consider the length of the channel in $\mathrm{cm}$ and the following domain

$$
\Omega=\left[\begin{array}{ll}
0 & 29
\end{array}\right] \times\left[\begin{array}{ll}
0 & 5
\end{array}\right] \backslash\left[\begin{array}{ll}
2.5 & 26.5
\end{array}\right] \times\left[\begin{array}{ll}
2.25 & 2.75
\end{array}\right]
$$

To include wall boundaries, we insert ghost particles with distance $l / 2$ at the boundaries with orientations parallel to the respective wall. The interaction potential is given by equation (4.2). For the interaction of the boundary particles with the inner particles, we increase the value of $\epsilon_{0}$ by a factor of 10 . For the surrounding fluid we us the results of the CFD simulation as described in Section 3. As mentionend before, to use the resulting 3D velocity field in the two-dimensional set up, we choose the time-averaged velocity field of the middle plane in $z$-direction (compare Figure 6).

Then we set the following parameters according to the experiment

$$
l=0.49 \mathrm{~cm}, \quad d=0.25 \mathrm{~cm}, \quad T=10 \mathrm{~s} .
$$




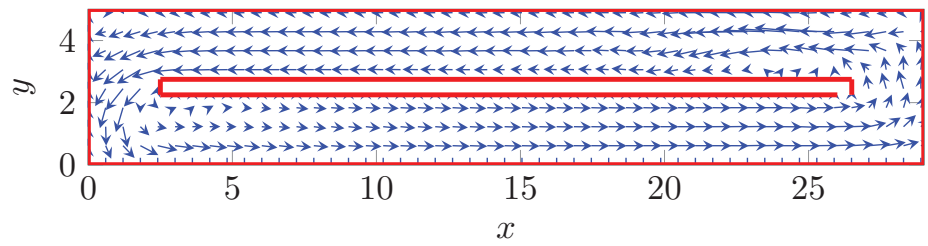

FiguRE 6. Experiment: velocity field of the surrounding fluid is given by the blue arrows, and the wall boundaries are indicated by the red line.

The mass $m$ and moment of inertia $I_{c}$ for the ellipsoidal particles are given by

$$
\begin{aligned}
& m=\rho V=\rho \frac{4}{3} \pi L D^{2}=0.97 \mathrm{~g}, \\
& I_{c}=\frac{m}{5}\left(L^{2}+D^{2}\right)=0.25 \mathrm{gcm}^{2} .
\end{aligned}
$$

The friction parameters for translational motion $\gamma_{t}$ and rotational motion $\gamma_{r}$ are only given in the direction of the main axis of the particle $[11,4]$. They have the following form

$$
\begin{aligned}
T_{t}^{L} & =16 \pi \bar{\eta} \frac{L^{2}-D^{2}}{\left(2 L^{2}-D^{2}\right) S-2 L}, \\
T_{t}^{D} & =32 \pi \bar{\eta} \frac{L^{2}-D^{2}}{\left(2 L^{2}-3 D^{2}\right) S-2 L}, \\
T_{r}^{L} & =\frac{32}{3} \pi \bar{\eta} \frac{\left(L^{2}-D^{2}\right) D^{2}}{2 L-D^{2} S} \\
T_{r}^{D} & =\frac{32}{3} \pi \bar{\eta} \frac{L^{4}-D^{4}}{\left(2 L^{2}-D^{2}\right) S-2 L},
\end{aligned}
$$

where

$$
S=\frac{2}{\sqrt{L^{2}-D^{2}}} \log \left(\frac{L+\sqrt{L^{2}-D^{2}}}{D}\right),
$$

and $\bar{\eta}$ is the viscosity of the fluid.

To get the correct friction parameters for our model, we have to divide $T_{t}^{L}$ and $T_{t}^{D}$ by the mass $m$ and $T_{r}^{L}$ and $T_{r}^{D}$ by the moment of inertia $I_{c}$. Furthermore, we use the dynamic viscosity of water which is given by $\bar{\eta}=0.001 \mathrm{~g} / \mathrm{cms}$. Then we get

$$
\gamma_{t}^{L}=0.111 / s, \quad \gamma_{t}^{D}=0.121 / s, \quad \gamma_{r}^{L}=0.21 / s, \quad \gamma_{r}^{D}=1.361 / s .
$$

Since our model has only one global friction parameter for the translation of the particles and one for the rotation of the particles, we have to do a best-fit approximation. In this case, we are mostly interested in the angular distribution of the particles. Therefore we take fixed values of $\gamma$ and $A$, and fit the rotational parameters. For the translational friction parameter $\gamma$, we take the mean between $\gamma_{t}^{L}$ and $\gamma_{t}^{D}$ and assume no stochastic force. Hence, we set

$$
\gamma=0.1151 / s, \quad A=0 .
$$

For the interaction potential we choose a large strength $\epsilon_{0}=1000$, to prevent the particles from overlapping. The remaining parameters for the rotation are $\bar{\gamma}$ and $B$, which have to be fitted to the experimental data. 
4.2. Fitting Parameters. To find the best parameter values for $\bar{\gamma}$ and $B$, we simulate the experiment for several values of $\bar{\gamma}$ and $B$ and compare the resulting angular distributions with the angular distribution of the experimental data. Therefore we choose initially $N=25$ particles equally-distributed inside $\Omega$ with equally-distributed initial angular orientation. Since in the experiment the particles are already moving, when the camera detection starts, we simulate first up to time $T=5$ and use the configuration of the particles at $T=5$ as initial conditions for our simulation. In the experiment the particles are detected inside of the domain

$$
\Omega_{H}=\left[\begin{array}{ll}
2.5 & 26.5
\end{array}\right] \times\left[\begin{array}{ll}
0 & 5
\end{array}\right] \backslash[2.526 .5] \times[2.252 .75] .
$$

We simulate the particle movement for different parameters of $\bar{\gamma}$ and $B$ for 10 seconds, i.e. up to $T=20$. Then, we derive the angular distribution of the particles over the whole domain and for the whole simulation to get the distribution inside $\Omega_{H}$ from $T=5$ to $T=10$. Finally, we derive the different angular distribution histograms with $N_{H}=18$ bars. Since we cannot distinguish the head and tail of a particle, we count the number of particles inside a range of 10 degrees modulus 180 degrees. Let $h_{i}, i=1, \ldots, N_{H}$ denotes the $i$-th bar value of the angular distribution histogram of the simulation and $h_{i}^{e x p}, i=1, \ldots, N_{H}$, the $i$-th bar value of the angular distribution histogram obtained from the experimental data, respectively. Then, the relative error $e$ between for the histograms is given by

$$
e=\frac{\sum_{i=1}^{18}\left|h_{i}-h_{i}^{e x p}\right|}{\sum_{i=1}^{18}\left|h_{i}^{e x p}\right|} .
$$

Now we choose $\bar{\gamma}=\{0.2,0.24,0.28,0.32,0.36\}$ and $B=\{0,0.25,0.5,0.75,1,1.5,2\}$ and compute the relative $L^{1}$-error for all different combinations. The results are shown in Figure 7. This yields the best fit parameters $\bar{\gamma}=0.36$ and $B=0.5$. However, we note that the solution is not very sensitive for a larger range of parameters, see again Figure 7. We compute additionally the error of the standard deviation. With the experimental standard deviation of $\sigma^{e x p}=0.997$ and the standard deviation $\sigma=0.987$ obtained from the simulation with the best fitting parameters, we get

$$
e_{\sigma}=\frac{\left|\sigma^{\exp }-\sigma\right|}{\left|\sigma^{\exp }\right|}=0.01
$$

\section{Comparison}

In this section we compare the experimental results with the numerical simulation. First of all, we show the position of the particles at different times of the experiment. We observe that most of the particles are orientated longitudinal to the stream of the flow, and follow the surrounding fluid (see Figure 8). For the numerical simulation we have choosen the parameters $\bar{\gamma}=0.36$ and $B=0.5$ obtained in the previous section. We observe that the particles are mostly oriented longitudinal to the flow (see Figure 9). We note that the particles do not have the same position as in the experiment, since their initial conditions are not exactly the same. 


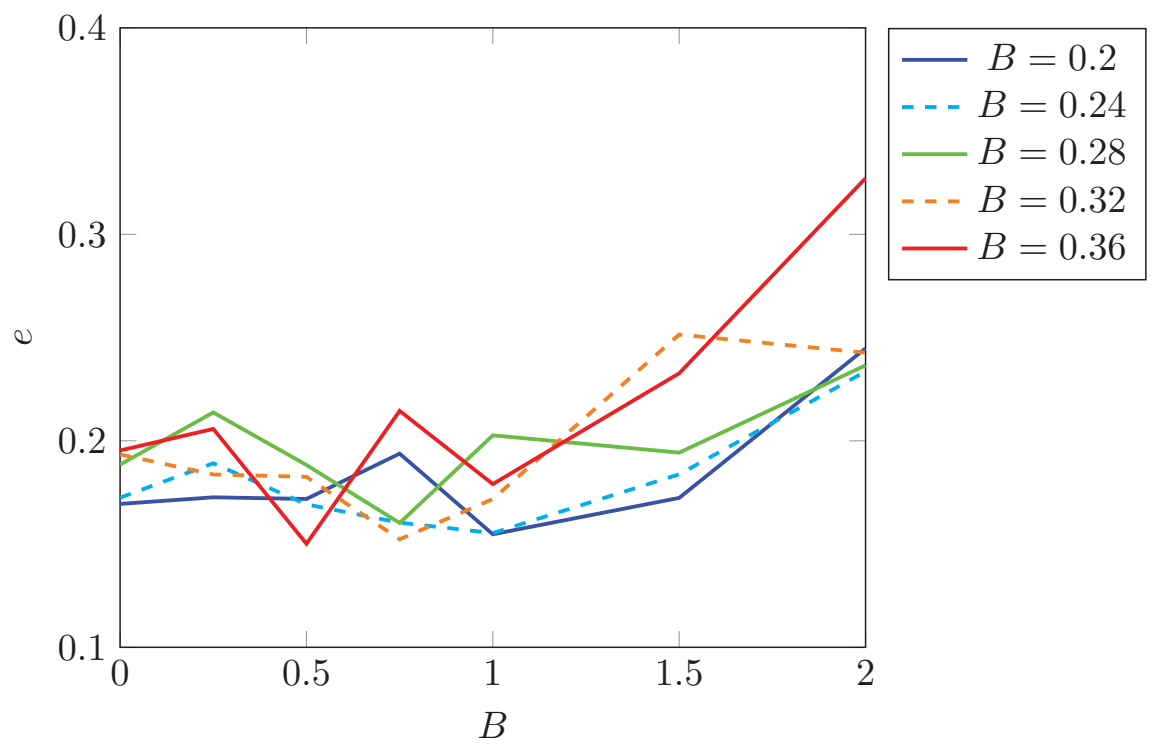

FIgURE 7. Relative $L^{1}$-error of the angular distribution for different $\bar{\gamma}$ and $B$.
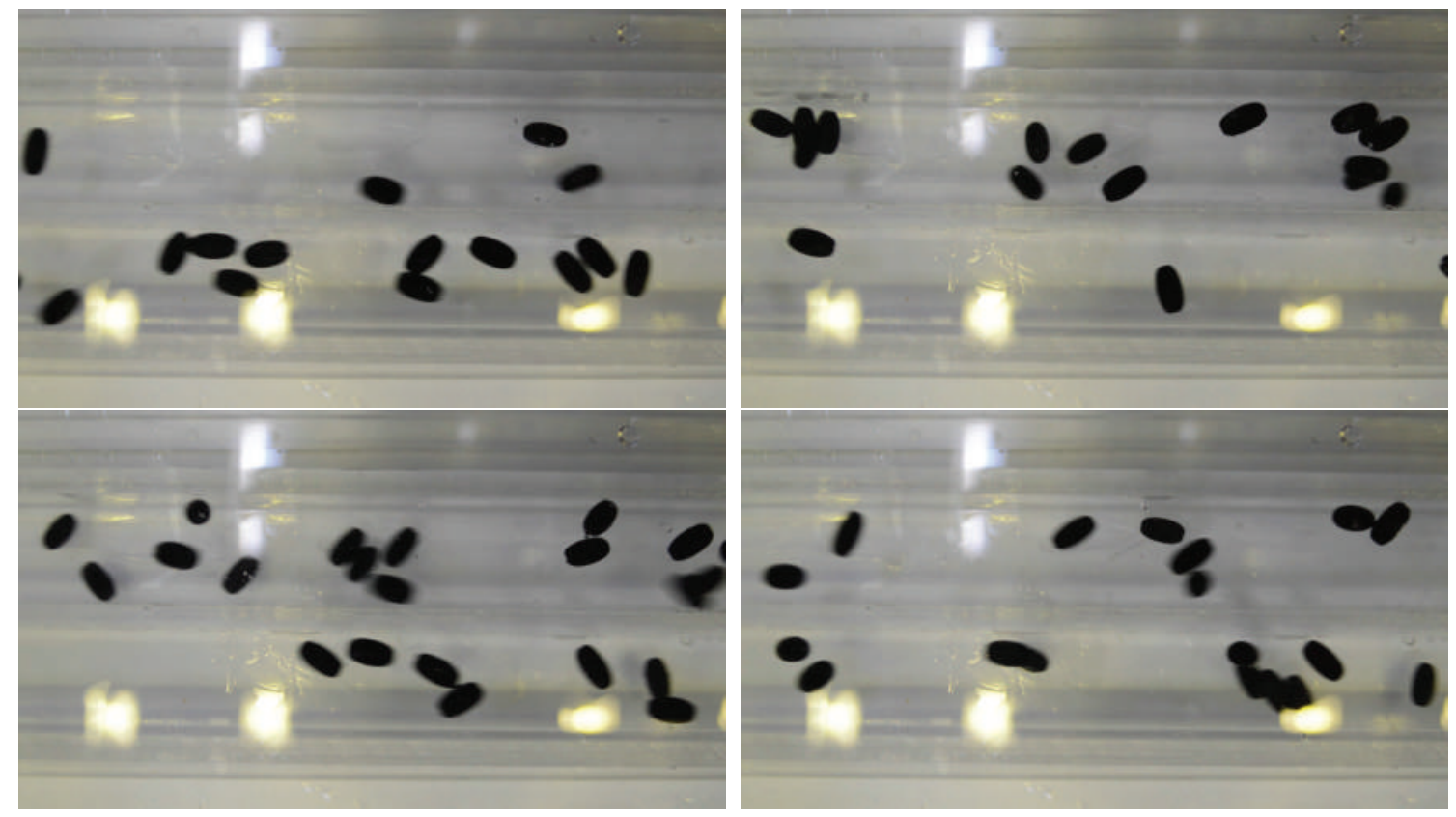

Figure 8. Pictures of the experiment with ellipsoidal particles (black particles) at times $\mathrm{t}=0,2.5,7.5,10$.

In Figure 10) we compare the histograms of the orientation of all particles. We observe that the orientation of the particles is similar for the experiment as for the simulation. The particles have a tendency to be orientated longitudinal to the stream of the flow, i.e. with angles concentrated around $\theta_{t}=0$ and $\theta_{t}=\pi$.

\section{Concluding Remarks}

We presented a basic model for interacting ellipsoids in fluid flow. Although our model has just two space dimensions, we observe a good agreement with the experimental data, 
Time $t=0.0$

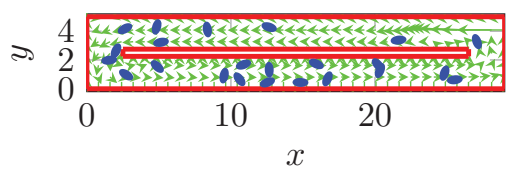

Time $\mathrm{t}=7.5$

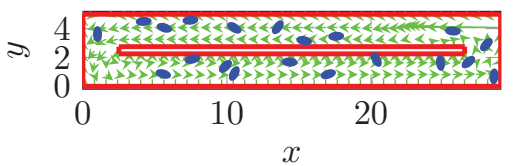

Time $t=2.5$

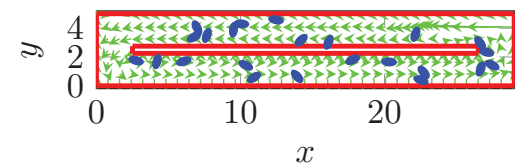

Time $\mathrm{t}=10.0$

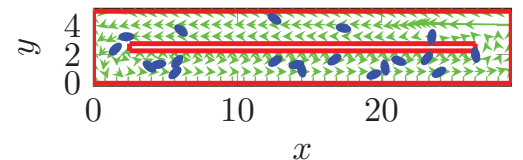

Figure 9. Numerical results of (4.1) of the experiment for different times. The wall boundaries are indicated by the red lines. The velocity field of the surrounding fluid is given by the green arrows, and the particles are marked by blue ellipses.

Angular distribution for the whole domain

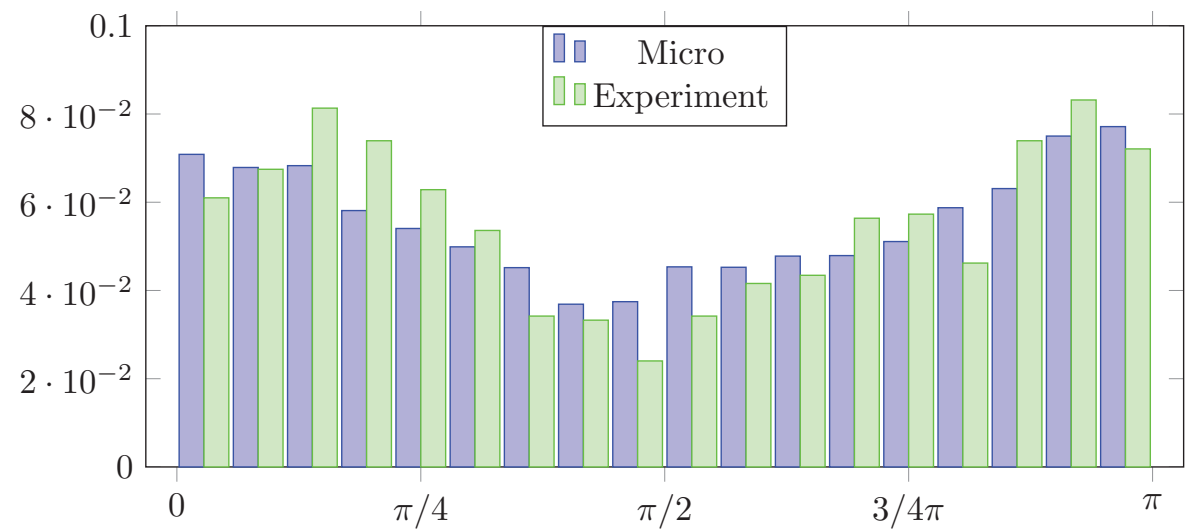

FIgURE 10. Angular distribution of the experiment for the experimental data and the numerical results of (4.1).

with a relative error of the standard derivation of $e_{\sigma}=0.01$, for the best fitting parameters. To fit the translational parameters, more experimental data are needed. Similarly, to add and validate more complex forces, further investigations and data are needed. Furthermore, if a large number of particles inside the channel are considered, a macroscopic description derived from the microscopic model investiagted here, see [1], could be used and compared to experimental data. This could enable large-scale simulations with moderate computational effort.

\section{ACKNOWLEDGMENT}

Funding by the Deutsche Forschungsgemeinschaft (DFG) within the RTG GrK 1932 "Stochastic Models for Innovations in the Engineering Sciences", project area P1, is gratefully acknowledged. 


\section{REFERENCES}

[1] R. Borsche, A. Klar, A. Meurer, O. Tse, Mean field models for interacting ellipsoidal particles, Computers and Mathematics with Applications, Vol. 72 (3), pp 704-719, 2016

[2] Y. Han, A. Alsayed, M. Nobili, J. Zhang, T. C. Lubensky, A. G. Yodh, Brownian motion of an ellipsoid Science, Vol. 314 (1), pp 626-630, 2006

[3] D. J. Cleaver, C. M. Care, M. P. Allen, M. P. Neal, Extension and gen- eralization of the gay-berne potential, Physical Review E, Vol. 54 (1), pp 559, 1996

[4] T. Norisuye, M. Motowoka, H. Fujita, Wormlike chains near the rod limit: translational friction coefficien, Macromolecules, Vol. 12 (2), pp 320-323, 1979

[5] G. B. Jeffery, The motion of ellipsoidal particles immersed in a viscous fluid, Proceedings of the Royal Society of London. Series A, Vol. 102 (715), pp 161-179, 1992

[6] J. Gay, B. Berne, Modification of the overlap potential to mimic a linear site-site potential, The Journal of Chemical Physics, Vol. 74 (6), pp 3316-3319, 1981

[7] J. Walter, O. Gonzalez, J. Maddocks, On the stochastic modeling of rigid body systems with application to polymer dynamics, Multiscale Modeling ES Simulation 8 (3) pp 1018-1053, 2010

[8] J. W. Perram, J. Rasmussen, E. Præstgaard, J. L. Lebowitz, Ellipsoid contact potential: Theory and relation to overlap potentials, Physical Review E. Vol. 54 (6), pp 6565, 1996

[9] R. Everaers, M. Ejtehadi, Interaction potentials for soft and hard ellipsoids, Physical Review E , 67 (4) pp 041710, 2003

[10] R. Berardi, C. Fava, C. Zannoni, A generalized gay-berne intermolecular potential for biaxial particles, Chemical Physics Letters, 236 (4), pp 462-468, 1995

[11] X. Sun, T. Lin, J. D. Gezelter, Langevin dynamics for rigid bodies of arbitrary shape, The Journal of chemical physics, Vol. 128 (23), pp 234107, 2008

[12] M. Junk, R. Illner, A new derivation of jeffery's equation, Journal of Mathematical Fluid Mechanics, Vol. 9 (4), pp 455-488, 2007

[13] J. Gay, B. Berne, Modification of the overlap potential to mimic a linear site-site potential, The Journal of Chemical Physics, Vol. 74 (6),pp 3316-3319, 1981

[14] M. Tavakol, O. Abouali, M. Yaghoubi, G. Ahmadi, Dispersion and de- position of ellipsoidal particles in a fully developed laminar pipe flow using non-creeping formulations for hydrodynamic forces and torques, International Journal of Multiphase Flow, Vol. 75, pp 54-67, 2015

[15] W. Coffey, Y. P. Kalmykov, J. T. Waldron, The Langevin equation: with applications in physics, chemistry, and electrical engineering, Vol. 10, World Scientific, Singapore, 1996

[16] ImageJ, version 1.49, Wayne Rasband, National Institutes of Health, Bethesda, Maryland, USA, 2015

[17] S. Senchenko, H. J. Keh, Slipping stokes flow around a slightly deformed sphere, Physics of Fluids, 18 (8), pp 88104, 2006, doi:10.1063/1.2337666

[18] Z. Y. Zhou, D. Pinson, R. P. Zou, A. B. Yu, Discrete particle simulation of gas fluidization of ellipsoidal particles, Chemical Engineering Science, Vol. 66 (23), pp 6128-6145, 2011, doi:10.1016/j.ces.2011.08.041

[19] J. M. Ottino, D. V. Khakhar, Fundamental research in heaping, mix- ing, and segregation of granular materials: challenges and perspectives, Powder Technology, Vol. 121 (2), pp 117-122, 2001

[20] H. A. Jakobsen, H. Lindborg, C. A. Dorao, Modeling of bubble column reactors: Progress and limitations, Industrial \& Engineering Chemistry Research, Vol. 44 (14), pp 5107-5151, 2005, doi:10.1021/ie049447x. 
